\title{
Multiple splitting of droplets using multi-furcating microfluidic channels
}

\author{
Cite as: Biomicrofluidics 13, 024112 (2019); doi: 10.1063/1.5086716 \\ Submitted: 22 December 2018 . Accepted: 14 April 2019. \\ Published Online: 26 April 2019

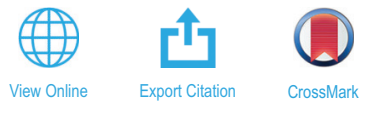

Zida Li, ${ }^{1, a)}$ (D) Luoquan $\mathrm{Li}^{2}{ }^{(1 D}$ Meixiang Liao, ${ }^{2}$ Liqun $\mathrm{He}^{2}$ (D) and Ping $\mathrm{Wu}^{2,3,4, a), b)}$

\begin{abstract}
AFFILIATIONS
${ }^{1}$ Department of Biomedical Engineering, School of Medicine, Shenzhen University, Shenzhen 518060, China

${ }^{2}$ Department of Thermal Science and Energy Engineering, University of Science and Technology of China, Hefei 230027, China

${ }^{3}$ BGI-Shenzhen, Shenzhen 518083, China

${ }^{4}$ China National GeneBank, BGI-Shenzhen, Shenzhen 518120, China
\end{abstract}

a) Authors to whom correspondence should be addressed: zidali@szu.edu.ch and wuping@directgenomics.com

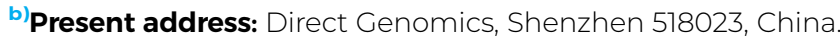

\begin{abstract}
Removing volumes from droplets is a challenging but critical step in many droplet-based applications. Geometry-mediated droplet splitting has the potential to reliably divide droplets and thus facilitate the implementation of this step. In this paper, we report the design of multifurcating microfluidic channels for efficient droplet splitting. We studied the splitting regimes as the size of the mother droplets varied and investigated the dependence of the transition between splitting regimes on the capillary number and the dimensionless droplet length. We found that the results obtained with our device agreed with the reported dimensionless analysis law in T-junctions. We further investigated the effect of channel lengths on the volume allocation in branch channels and achieved droplet splitting with various splitting ratios. This study proposed an efficient on-demand droplet splitting method and the findings could potentially be applied in washing steps in dropletbased biological assays or assays that require aliquot.
\end{abstract}

Published under license by AIP Publishing. https://doi.org/10.1063/1.5086716

\section{INTRODUCTION}

Homogeneous nanoliter/picoliter droplets have great applications in fields such as food industry, ${ }^{1}$ cosmetics, ${ }^{2}$ single cell RNA/ DNA sequencing, ${ }^{3}$ droplet digital polymerase chain reaction (ddPCR), ${ }^{4,5}$ nanoparticle synthesis, ${ }^{6}$ drug delivery, ${ }^{7}$ and drug discovery. ${ }^{8}$ These droplets offered a platform for precise control over reagent quantity, which is critical for reaction reproducibility and reliability. Additionally, cells and objects can be encapsulated in the droplets, providing a means to manipulate single cells. ${ }^{9,10}$

Traditionally, droplets are prepared by mechanical agitation. Despite its wide applications, this method suffered from poor droplet monodispersity, leading to compromised process controllability. Leveraging the strict laminar flow and Plateau-Rayleigh instability, microfluidics has been proved as a powerful tool for the generation of highly monodispersed droplets, typically with a coefficient of variation in droplet size between $1 \%$ and $3 \% .{ }^{11}$ In addition, the throughput of droplet generation is high: hundreds to thousands of droplets can be generated each second, depending on the flow conditions. Many chemical and biological applications have been implemented in droplet-based microfluidics, such as drug screening, enzyme revolution, particle synthesis, material synthesis, and single cell sequencing. ${ }^{9,10,12,13}$

In a typical application of droplet-based microfluidics, chemicals or objects are encapsulated within individual droplets during droplet formation. Many downstream techniques have been developed to achieve specific droplet manipulation. For example, droplet sorting techniques were developed for encapsulation enrichment, ${ }^{14}$ droplet merging for large volume reagent exposure or object contact, ${ }^{15}$ and pico-injection for small volume reagent addition. ${ }^{16}$ These techniques served as powerful tools for downstream droplet manipulation. Nevertheless, most of these techniques focused on volume addition. Volume removal, on the other hand, is equally important in many application scenarios. For example, many biological assays, such as immunoassays, incorporate one or more washing steps, requiring both reagent addition and removal. When performed in droplets, these assays can be implemented by encapsulating 
microbeads, which serve as attaching substrates. By adding and removing reagents from the droplets, washing steps can be implemented. Therefore, droplet splitting techniques for efficient volume removal are critical for these applications.

Many studies have been reported to achieve droplet splitting. Depending on the techniques involved, these methods could be categorized into active and passive splitting. Active splitting methods break droplets by introducing external force, leading to splitting with good controllability. For example, static electric field was reported as a robust tool to facilitate droplet bi-splitting. ${ }^{17}$ Liquid wettability of dielectric surfaces can be controlled by electric potential; based on the principle of electrowetting on dielectric (EWOD), droplet splitting as well as transporting and merging have been demonstrated. ${ }^{18}$ Using a multilayer microfluidic chip with a pneumatic control layer, researchers proposed droplet splitting with pneumatic valves. ${ }^{19}$ Splitting based on thermocapillary ${ }^{20}$ and acoustic waves ${ }^{21,22}$ were also shown as powerful tools. Despite the great applications these methods have shown, they were featured with extra physical fields, thus limiting their applications in practice. In contrast, passive splitting mechanisms did not require external forces and broke droplets with deliberate fluidic designs. With carefully designed $\mathrm{T}$-junctions in microfluidic devices, passive droplets splitting mediated by geometry and the resultant hydraulic resistance were reported. ${ }^{23,24}$ Based on this channel design, breakup dynamics in asymmetric T-junctions, ${ }^{25}$ junctions with arbitrary angles, ${ }^{26}$ and in flow past obstacles ${ }^{27}$ were investigated. To minimize the adverse effect of surface roughness on splitting, stepwise bifurcating channels in bilayer microfluidic devices were proposed. ${ }^{28-30}$

These methods produced daughter droplets with designed sizes and good reproducibility. Nevertheless, such binary splitting has limited efficiency for applications where multiple aliquoting and thus multiple splitting are necessary. To address this problem, herein we report a simple yet robust droplet splitting mechanism which offers tunable amount and splitting ratio among the daughter droplets. In particular, we adopted multi-furcating channels to break up the mother droplets and direct the daughter droplets. When the mother droplets came to the furcating region, shear stress and the notches of the branch channels deformed them into narrow rod-like shapes, facilitating the breakup into daughter droplets. We investigated the effect of mother droplet sizes and found that as the droplet size increased, the droplet splitting fell in different regimes, including no-splitting, nonplug splitting, and plug splitting regime. We then analyzed the transition from no-splitting to one-to-three splitting and from one-to-three splitting to one-to-five splitting using dimensionless analysis. The results showed that the critical capillary number for initial droplet breakup scaled with dimensionless droplet length while further transition had a weak dependence on the capillary number. We further investigated the effect of flow resistances on splitting results. Our results showed that by adjusting channel lengths, the splitting results could be tuned. This droplet splitting method holds great potential as a versatile tool in droplet-based biological assays which require washing or sample splitting.

\section{MATERIALS AND METHODS}

Microfluidic devices were fabricated by standard photolithography and polydimethylsiloxane (PDMS) replica molding. Briefly,
SU8 (2030, MicroChem Corp.) was spin-coated on a silicon wafer, typically with a thickness of $37 \mu \mathrm{m}$, before it was exposed to UV light and developed. The fabricated silicon wafer with an SU8 structure was then silanized before it was used for PDMS (Sylgard 184, Dow Chemical Company; 10:1 base-to-hardener ratio) replica molding. The cured PDMS was then cut and peeled off from the silicon wafer. Holes with diameters of $1 \mathrm{~mm}$ were punched using biopsy punches. The PDMS slab was then bonded with a glass slide after oxygen plasma treatment (PDC-32G-2, Harrick Plasma) for $1 \mathrm{~min}$ and baked for $5 \mathrm{~min}$. The dimension of the fabricated channels possessed good consistency, with a measured width of $184 \mu \mathrm{m}$, a variation of $4.28 \%$ among widths of branch channels, and relatively smooth surfaces (Fig. S1 in the supplementary material).

Chemical reagents were loaded in $1 \mathrm{ml}$ glass syringes (Hamilton Company) and infused into the microfluidic chips by syringe pumps (NE-1200, New Era Pump Systems Inc.) via polytetrafluoroethylene (PTFE) tubing (1522, IDEX Health \& Science LLC). An inverted microscope (IX73, Olympus Corp.) coupled with a high speed camera was used to observe and image the experiments.

Mineral oil (M3516-1L, Sigma Aldrich) with a dynamic viscosity of $0.03 \mathrm{~Pa}$ s was used as the continuous phase, with $0.05 \%$ Triton X-100 (T9284, Sigma Aldrich) and 2\% ABIL EM90 supplemented as the surfactant. De-ionized water was used as the disperse phase. The interfacial tension of the selected system was $46.28 \mathrm{mN} / \mathrm{m}$, as measured with a tensiometer. ${ }^{31}$ Diameters of the generated droplets were measured using ImageJ (National Institute of Health, USA).

\section{RESULTS}

\section{Overview of the splitting setup and demonstration of the splitting performance}

Mother droplets with uniform size were generated with T-junctions, as shown in Fig. S2 in the supplementary material, typically with a coefficient of variation of less than $5 \%$ in droplet diameter. ${ }^{31}$ A cross junction was designed at the downstream to break up the mother droplets and direct the daughter droplets toward designated outlets [Fig. 1(a)]. Upon arriving at the cross junction, due to pressure drop and shear stress, the droplet was deformed into a rod shape with a significantly reduced diameter. The area around the notch between adjacent channels has elevated pressure, which squeezes the droplet and leads to a locally thinned rod diameter. Eventually, the droplet breaks up at the loci of the droplet close to the notches.

With different channel designs, splitting of droplets into two, three, and five daughter droplets were achieved, respectively [Fig. 1(b)]. Daughter droplets showed symmetry along the axis of the main channel: droplets moving toward north and south displayed similar droplet sizes, and those moving toward northeast and southeast displayed similar sizes. This observation agreed with the symmetry of the channel designs. Among each branch channel, the droplets showed high uniformity, with coefficients of variation in the droplet diameters typically less than $5 \%$.

The splitting results largely depended on the pressure distribution of the flow field, which was the coupled result of a few parameters including flow rates, viscosities of the fluids, interfacial tension, size of the mother droplet, cross junction geometry, 
(a)

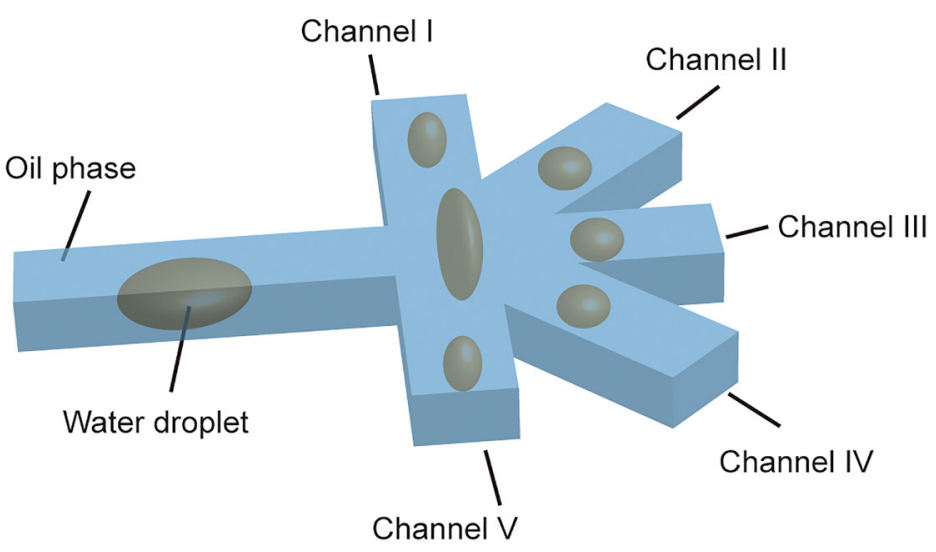

(b)

1 into 2

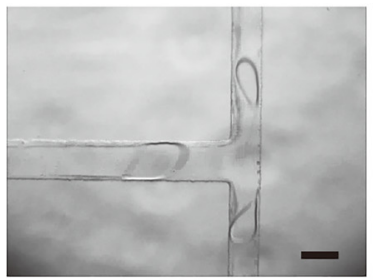

1 into 3

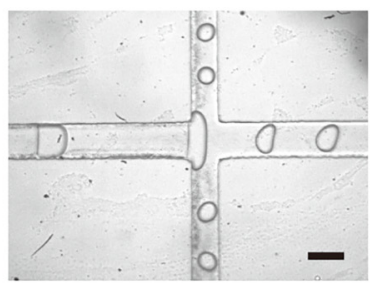

1 into 5
FIG. 1. (a) Schematic showing the droplet spliting setup. A droplet coming downstream to the junction splits into a few daughter droplets. (b) Representative images showing droplets split into two, three, and five daughter droplets, as indicated. Scale bars, $200 \mu \mathrm{m}$. Flow rates of the continuous phase and disperse phase were 6 and 1,10 and 1 , and 2 and $0.5 \mu \mathrm{l} / \mathrm{min}$, respectively. and flow resistance of the branch channels. Size of the mother droplet, in particular, is dependent on the flow rates, fluid viscosities, and interfacial tension. To simplify the study, we focused the study on the effect of size of the mother droplets and flow resistance of the branch channels.

\section{The effect of the size of the mother droplets on the resultant daughter droplets}

The size of the mother droplet affects its shape at the junction, critically affecting the splitting results. The size of the mother droplets can be adjusted by tuning the flow rates of the continuous phase (oil) and disperse phase (water). ${ }^{31-33}$ We investigated the effect of mother droplet size on splitting in channels with one-into-five star-shaped junctions by varying the mother droplet size from 50 to $350 \mu \mathrm{m}$. Results showed that when the mother droplet was small, typically with a diameter below $70 \mu \mathrm{m}$, it was slightly deformed at the junction but entered only Channel III [designated in Fig. 1(a)] with no splitting, as shown in Fig. 2. This observation was likely due to that small droplet experienced small pressure drop and thus less deformation. As the length did not exceed the circumference, the droplet did not break up. We named this the no-splitting regime.

As the droplet size increased, the two channels adjacent to Channel III, namely, Channel II and Channel IV, started to receive daughter droplets. When the droplet diameter reached about $100 \mu \mathrm{m}$, the mother droplet split into five daughter droplets, directed into five

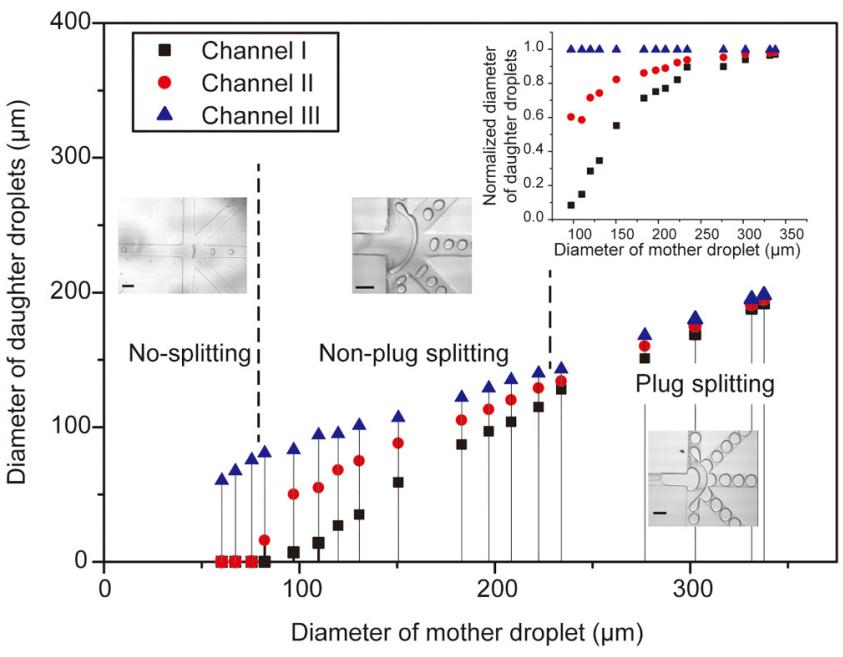

FIG. 2. Droplet splitting fell into three regimes, namely, nonsplitting, nonplug splitting, and plug splitting, as the diameter of the mother droplet increased. In the plug splitting regime, daughter droplets showed similar sizes. Channel names were designated as in Fig. 1(a). Scale bars, $200 \mu \mathrm{m}$. The flow rate of the continuous phase was fixed at $2 \mu \mathrm{l} / \mathrm{min}$ while that of the disperse phase varied from 0.1 to $1 \mu \mathrm{l} / \mathrm{min}$. Tabulated data are shown in Table S2 in the supplementary material. Inset: Normalized diameters of daughter droplets, which were the ratios of droplet diameters to those of Channel III, plotted as a function of the diameters of mother droplets. 


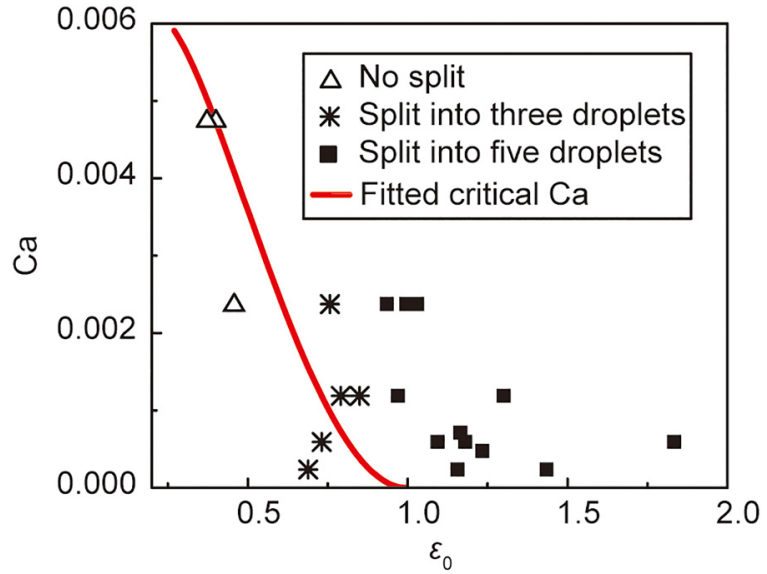

FIG. 3. Phase diagram of the droplet split regimes with the capillary number $\mathrm{Ca}$ and dimensionless initial droplet length $\varepsilon_{0}$ as the variables. Critical conditions for the transition from no split to split into three droplets are plotted based on curve fitting.

branches, respectively. Droplets in mirrored channels, namely, Channel I \& V and Channel II \& IV, typically had similar droplet sizes, due to the symmetry of the channel geometry. At this stage, the diameters of the daughter droplets in Channel III were always the largest among those of the five channels. However, the difference in the droplet sizes between Channel III and other channels was narrowed as the diameter of the mother droplets increased. When the diameters of the mother droplet reached about $230 \mu \mathrm{m}$, the daughter droplets had almost the same diameters, as shown in the inset of Fig. 2. We noticed that at this stage, mother droplets obstructed the continuous phase and formed a plug flow, and the daughter droplets also covered up the whole cross section of the branch channels, forming plug flows as well. As such, we named the former as the nonplug splitting regime and the latter as the plug splitting regime, as indicated in Fig. 2. The observation of equal-sized daughter droplets in plug splitting regime was likely due to that the volume flow rates in the branch channels were equal. Similar results were also observed in one-into-three junction structures (Fig. S3 in the supplementary material).

\section{Analysis of the breakup phenomena}

To tease out the deterministic factors of the splitting, we closely examined the transition from the no-split regime to the nonplug splitting regime. Intuitively, the splitting depended on the shape of the mother droplet while being discharged into the junction and its shape was mainly determined by its size, viscous stress, and interfacial tension. Thus, we took the capillary number $\mathrm{Ca}$, which represents the ratio between viscous stress and surface tension, and the droplet's initial dimensionless length $\varepsilon_{0}$, defined as the ratio of droplet's initial length $l_{0}$ to its initial circumference $\pi w_{0}$, as the variables and plotted the phase diagram of splitting regime ${ }^{24}$ (Fig. 3). Capillary is defined by $\mathrm{Ca}=\eta v / \gamma$ where $\eta$ is the viscosity of the continuous phase, $v$ is the flow velocity, and $\gamma$ is the interfacial tension. The dependence of the critical $\mathrm{Ca}, \mathrm{Ca} a_{c r}$ for droplet breakup on droplet size follows ${ }^{24,30} C a_{c r} \propto\left(l_{e}-l_{0}\right)^{2}$, where $l_{e}$ is the stretched droplet length. Plateau-Rayleigh instability predicts $l_{e}=\pi w_{e}$ at the stability limit, where $w_{e}$ is the stretch droplet width. Volume conservation gives $l_{0} w_{0}^{2}=l_{e} w_{e}^{2}$. Combining these three equations, we get

$$
C a_{c r}=\alpha \varepsilon_{0}^{2}\left(1 / \varepsilon_{0}^{2 / 3}-1\right)^{2},
$$

where $\alpha$ is a dimensionless constant depending on fluid viscosity and channel geometry. With our experimental data, $\alpha$ was fitted as 0.042 and the fitted curve is shown in Fig. 3. This analysis method, though proposed in studies of droplet splitting at T-junctions, agreed reasonably well with the experimental data in our case. This agreement was likely due to that while the droplets were small, they did not collide with the branch notches and the breakup was mainly governed by Plateau-Rayleigh instability, similar to breakup in T-junctions.

Within the splitting regime, the droplet initially split into three droplets. As the droplet size increased, it transited into a state where five droplets were generated. The results showed that this transition had much weaker dependence on $C a$ as compared to $\varepsilon_{0}$, as shown in Fig. 3. The Pearson correlation coefficient between splitting regime and $\mathrm{Ca}$ was -0.25 with a $\mathrm{P}$-value of 0.31 , while that between splitting regime and $\varepsilon_{0}$ was 0.77 with a P-value of (a)

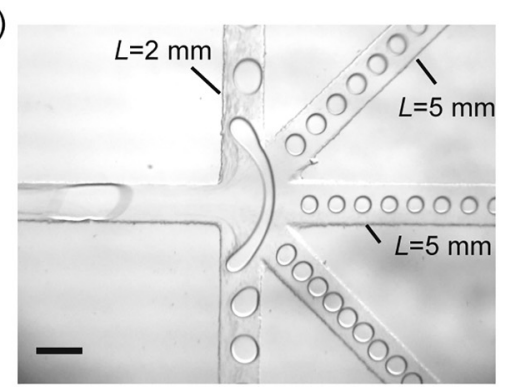

(b)

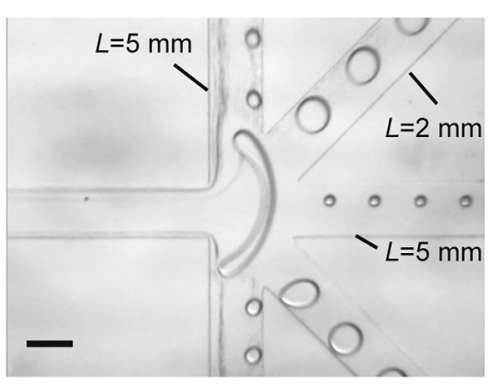

(c)

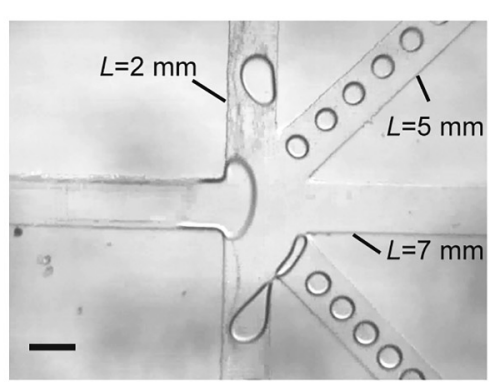

FIG. 4. Effect of channel length on the resultant daughter droplets. (a)-(c) Micrographs showing the droplet splitting with channel lengths as indicated. Upper and lower halves were symmetric. Lengths of the branches decided the flow resistances, leading different flow rates and thus droplet sizes. Flow rates of the continuous and disperse phase were 2 and $0.5 \mu \mathrm{l} / \mathrm{min}$, respectively. Scale bars, $200 \mu \mathrm{m}$. 
(a)

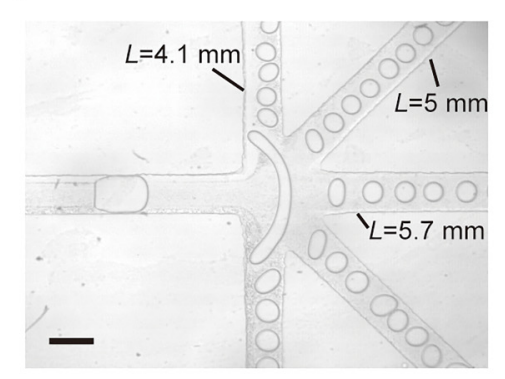

(b)

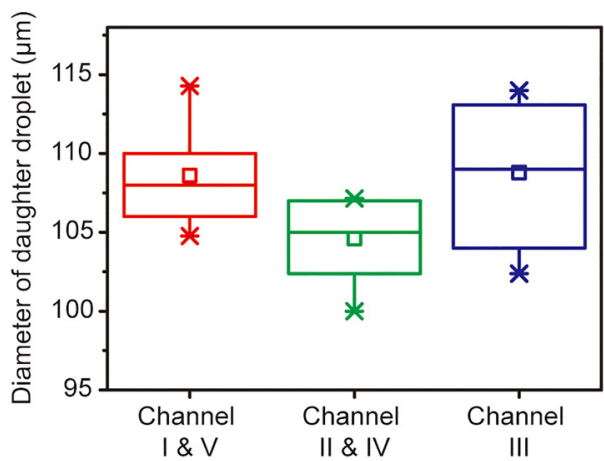

FIG. 5. Generation of droplets with the same sizes in all channels by tuning the channel lengths. (a) Microscopic images showing equal-sized droplets generated in each branch channels. Scale bar, $200 \mu \mathrm{m}$. (b) Box plot of the diameter of the daughter droplet in each branch channels.
0.0002. A large P-value indicated an insignificant correlation. It suggested that the transition between one-to-three splitting and one-to-five splitting was mainly governed by the size of the mother droplet, likely since the breakup was mainly mediated by the droplet colliding on the notches.

\section{Effect of channel lengths on splitting ratio in the nonplug splitting regime}

Though in the plug splitting regime the splitting ratio is relatively predictable, mother droplets would split evenly into each branch channel; in the nonplug splitting regime, the splitting ratio is more diverse. According to the Hagen-Poiseuille law, the pressure drop, $\Delta P$, caused by viscous dissipation in laminar flow follows $\Delta P=8 \mu L Q / \pi d^{4}$, where $\mu$ is the dynamic viscosity, $L$ is the channel length, $Q$ is the volumetric flow rate, and $d$ is the hydraulic diameter. Thus, the flow resistance, $R$, can be determined by $R=8 \mu L / \pi d^{4}$. Since the splitting process depended on the flow field and thus flow resistances of the branch channels, we asked whether the sizes of the daughter droplets could be controlled by varying the lengths of the branch channels. Using one-into-five junction designs, we varied the length of the side channels. Decreasing the lengths of the side channels decreased their flow resistance, resulting in a smaller pressure drop between the main channel and side branches. Consequently, the daughter droplets split into these channels would have large sizes.

Indeed, when Channel I \& V had a length of $2 \mathrm{~mm}$ and all other channels had lengths of $5 \mathrm{~mm}$, the diameters of the daughter droplets in Channel I \& V were significantly larger than those in other channels, as shown in Fig. 4(a). Similarly, when Channel II \& IV were $2 \mathrm{~mm}$ long and keeping Channel I \& V and Channel III at $5 \mathrm{~mm}$ long, the diameters of the daughter droplets in Channel II and IV were much larger than Channel I \& V and Channel III, as shown in Fig. 4(b). Furthermore, by increasing the resistance of Channel III and decreasing that of Channel I \& V, we were able to avoid daughter droplets entering Channel III. For example, when the lengths of Channel I \& V, Channel II \& IV, and Channel III were 2, 5, and $7 \mathrm{~mm}$, respectively, we observed larger daughter droplets in Channel I and V but daughter droplets were absent in Channel III.

These results showed that by adjusting the length of the branch channels, we could tune the splitting ratio. Since droplet microfluidics is generally nonplug flow and droplet splitting usually lies in the nonplug splitting regime, this observation could have profound applications. We investigated whether this device could achieve on-demand droplet splitting with carefully designed channel length. Specifically, we studied the parameters that could generate equal-sized droplets in the five channels by adjusting the lengths of Channel I \& V and Channel III while keeping Channel II \& IV at $5 \mathrm{~mm}$. As we set the lengths of Channel I \& V, Channel II \& IV, and Channel III as $4.1,5$, and $5.7 \mathrm{~mm}$, respectively, the diameters of the resultant daughter droplets were very close (Fig. 5). The measured diameters of the droplets in Channel I \& V, Channel II \& IV, and Channel III were 108.6 $\pm 3.1,104.6 \pm 2.6$, and $108.8 \pm 4.3 \mu \mathrm{m}$, respectively. These results showed that by a careful design, on-demand desirable droplet splitting ratios could be achieved.

\section{CONCLUSION}

In this paper, we reported the observation of droplet splitting in microfluidic channels. In particular, we fabricated furcating channels and split the mother droplet into five daughter droplets in a consistent manner. We investigated the effect of the sizes of mother droplets and found that as the size of the mother droplet increased, the splitting fell into three regimes, namely, nonsplitting, nonplug splitting, and plug splitting regimes. We analyzed the experimental results with the dimensionless law and confirmed the dependence between the critical capillary number for droplet breakup and the dimensionless droplet length. We further studied the effect of flow resistance in the branch channels, particularly the lengths of branch channels, on the droplet splitting. We found the channel length arrangement that could lead to equal-sized daughter droplets formation. This study provided a proof-of-concept method for volume splitting in droplet-based applications where aliquot or washing is difficult to implement.

\section{SUPPLEMENTARY MATERIAL}

See the supplementary material for tabulated data and additional figures on channel characterization, droplet generation, and splitting in one-to-three junctions. 


\section{ACKNOWLEDGMENTS}

This work is supported by the National Natural Science Foundation of China (NNSFC) (Nos. NSFC 31500694 and NSFC 31670866).

\section{NOMENCLATURE}

$\mathrm{Ca}$ Capillary number

$l_{0} \quad$ Initial droplet length

$l_{e} \quad$ Stretched droplet length

$v \quad$ Velocity of the flow

$w_{0} \quad$ Initial droplet width

$w_{e} \quad$ Stretched droplet width

\section{Greek}

$\varepsilon_{0} \quad$ Dimensionless initial droplet length

$\eta \quad$ Dynamic viscosity of the continuous phase

$\gamma$ Interfacial tension

\section{REFERENCES}

${ }^{1}$ F. L. Tchuenboumagaia, I. T. Norton, and P. W. Cox, "Hydrophobins stabilised air-filled emulsions for the food industry," Food Hydrocoll. 23(7), 1877-1885 (2009).

2J. Schreiber and K. H. Diec, "Crosslinked oil droplet-based cosmetic or pharmaceutical emulsions,” U.S. patent 7,811,594 (2010).

${ }^{3}$ E. Brouzes, M. Medkova, N. Savenelli, D. Marran, M. Twardowski, J. B. Hutchison, J. M. Rothberg, D. R. Link, N. Perrimon, and M. L. Samuels, "Droplet microfluidic technology for single-cell high-throughput screening," Proc. Natl. Acad. Sci. U.S.A. 106(34), 14195-14200 (2009).

${ }^{4}$ M. M. Kiss, L. Ortoleva-Donnelly, N. R. Beer, J. Warner, C. G. Bailey, B. W. Colston, J. M. Rothberg, D. R. Link, and J. H. Leamon, "High-throughput quantitative PCR in picoliter droplets,” Anal. Chem. 80(23), 8975 (2008).

${ }^{5}$ J. Chen, Z. Luo, L. Li, J. He, L. Li, J. Zhu, P. Wu, and L. He, "Capillary-based integrated digital PCR in picoliter droplets," Lab Chip 18(1), 412-421 (2018).

${ }^{6}$ L. H. Hung, K. M. Choi, W. Y. Tseng, Y. C. Tan, K. J. Shea, and A. P. Lee, "Alternating droplet generation and controlled dynamic droplet fusion in microfluidic device for CdS nanoparticle synthesis," Lab Chip 6(2), 174-178 (2006).

${ }^{\mathbf{7}}$ T. Gershanik, S. Benzeno, and S. Benita, "Interaction of a self-emulsifying lipid drug delivery system with the everted rat intestinal mucosa as a function of droplet size and surface charge," Pharm. Res. 15(6), 863-869 (1998).

${ }^{8}$ O. J. Dressler, R. M. Maceiczyk, S. I. Chang, and A. J. Demello, "Droplet-based microfluidics: Enabling impact on drug discovery,” J. Biomol. Screen. 19(4), 483 (2014).

${ }^{9}$ A. M. Klein, M. Linas, A. Ilke, T. Naren, V. Adrian, L. Victor, P. Leonid, D. A. Weitz, and M. W. Kirschner, "Droplet barcoding for single-cell transcriptomics applied to embryonic stem cells," Cell 161(5), 1187-1201 (2015).

${ }^{10}$ L. Mazutis, J. Gilbert, W. Lloyd Ung, D. A. Weitz, A. D. Griffiths, and J. A. Heyman, "Single-cell analysis and sorting using droplet-based microfluidics," Nat. Protoc. 8(5), 870-891 (2013).

${ }^{11}$ Z. Li, S. Y. Mak, A. Sauret, and H. C. Shum, "Syringe-pump-induced fluctuation in all-aqueous microfluidic system implications for flow rate accuracy," Lab Chip 14(4), 744-749 (2014).

${ }^{12}$ G. S. Du, J. Z. Pan, S. P. Zhao, Y. Zhu, J. M. den Toonder, and Q. Fang, "Cell-based drug combination screening with a microfluidic droplet array system,” Anal. Chem. 85(14), 6740-6747 (2013).
${ }^{13}$ R. Arayanarakool, L. Shui, S. W. Kengen, d. B. A. Van, and J. C. Eijkel, "Single-enzyme analysis in a droplet-based micro- and nanofluidic system," Lab Chip 13(10), 1955-1962 (2013).

${ }^{14}$ E. Koshimizu, S. Miyatake, N. Okamoto, M. Nakashima, Y. Tsurusaki, N. Miyake, H. Saitsu, and N. Matsumoto, "Performance comparison of benchtop next generation sequencers using microdroplet PCR-based enrichment for targeted sequencing in patients with autism spectrum disorder," PLoS One 8(9), e74167 (2013).

${ }^{15}$ B. J. Jin, Y. W. Kim, Y. Lee, and J. Y. Yoo, "Droplet merging in a straight microchannel using droplet size or viscosity difference," J. Micromech. Microeng. 20(3), 035003 (2010).

${ }^{16}$ B. O’Donovan, D. J. Eastburn, and A. R. Abate, "Electrode-free picoinjection of microfluidic drops," Lab Chip 12(20), 4029-4032 (2012).

${ }^{17}$ D. R. Link, E. Grasland-Mongrain, A. Duri, F. Sarrazin, Z. Cheng, G. Cristobal, M. Marquez, and D. A. Weitz, "Electric control of droplets in microfluidic devices,” Angew. Chem. 118(16), 2618-2622 (2006).

${ }^{18}$ S. K. Cho, H. Moon, and C.-J. Kim, "Creating, transporting, cutting, and merging liquid droplets by electrowetting-based actuation for digital microfluidic circuits," J. Microelectromech. Syst. 12(1), 70-80 (2003).

${ }^{19}$ J.-H. Choi, S.-K. Lee, J.-M. Lim, S.-M. Yang, and G.-R. Yi, "Designed pneumatic valve actuators for controlled droplet breakup and generation," Lab Chip 10(4), 456-461 (2010).

${ }^{20}$ Y.-F. Yap, S.-H. Tan, N.-T. Nguyen, S. M. S. Murshed, T.-N. Wong, and L. Yobas, "Thermally mediated control of liquid microdroplets at a bifurcation," J. Phys. D Appl. Phys. 42(6), 065503 (2009).

${ }^{21}$ J. H. Jung, G. Destgeer, B. Ha, J. Park, and H. J. Sung, “On-demand droplet splitting using surface acoustic waves," Lab Chip 16(17), 3235-3243 (2016).

22 J. Park, J. H. Jung, K. Park, G. Destgeer, H. Ahmed, R. Ahmad, and H. J. Sung, "On-demand acoustic droplet splitting and steering in a disposable microfluidic chip," Lab Chip 18(3), 422-432 (2018).

${ }^{23}$ A. R. Abate and D. A. Weitz, "Faster multiple emulsification with drop splitting," Lab Chip 11(11), 1911-1915 (2011).

${ }^{24}$ D. R. Link, S. L. Anna, D. A. Weitz, and H. A. Stone, "Geometrically mediated breakup of drops in microfluidic devices," Phys. Rev. Lett. 92(5), 054503 (2004).

${ }^{25}$ M. Samie, A. Salari, and M. B. Shafii, "Breakup of microdroplets in asymmetric T junctions," Phys. Rev. E 87(5), 053003 (2013).

${ }^{26}$ L. Ménétrier-Deremble and P. Tabeling, "Droplet breakup in microfluidic junctions of arbitrary angles,” Phys. Rev. E 74(3), 035303 (2006).

${ }^{\mathbf{2 7}}$ S. Protiere, M. Z. Bazant, D. A. Weitz, and H. A. Stone, "Droplet breakup in flow past an obstacle: A capillary instability due to permeability variations," Europhys. Lett. 92(5), 54002 (2010).

${ }^{\mathbf{2 8}}$ Y. Ren, K. S. Koh, J. K. Chin, J. Wang, C. Wen, and Y. Yan, "Droplet formation and fission in shear-thinning/Newtonian multiphase system using bilayer bifurcating microchannel," J. Heat Transf. 140(1), 012405 (2018).

${ }^{29}$ C. Lim, K. Koh, Y. Ren, J. Chin, Y. Shi, and Y. Yan, “Analysis of liquid-liquid droplets fission and encapsulation in single/two layer microfluidic devices fabricated by xurographic method," Micromachines 8(2), 49 (2017).

${ }^{30}$ Y. Ren, K. Koh, M. Yew, J. Chin, Y. Chan, and Y. Yan, "Droplet breakup dynamics in bi-layer bifurcating microchannel," Micromachines 9(2), 57 (2018).

${ }^{31} \mathrm{P}$. Wu, Y. Wang, Z. Luo, Y. Li, M. Li, and L. He, "A 3D easily-assembled Micro-Cross for droplet generation," Lab Chip 14(4), 795-798 (2014).

${ }^{32}$ T. Nisisako, T. Ando, and T. Hatsuzawa, "High-volume production of single and compound emulsions in a microfluidic parallelization arrangement coupled with coaxial annular world-to-chip interfaces," Lab Chip 12(18), 3426-35 (2012).

${ }^{33} \mathrm{P}$. Zhu and L. Wang, "Passive and active droplet generation with microfluidics: A review," Lab Chip 17(1), 34-75 (2016). 